\title{
Forward jets and forward $W$-boson production at hadron colliders
}

\section{J. R. Andersen and W.J. Stirling}

Institute for Particle Physics Phenomenology, University of Durham

Durham, DH1 3LE, U.K.

E-mail: 'Jeppe.Andersen@durham.ac.uk, W.J.Stirling@durham.ac.uk

\section{Del Duca*}

Istituto Nazionale di Fisica Nucleare, Sez. di Torino

via P. Giuria, 1 - 10125 Torino, Italy

E-mail: 'delduca@to.infn.it',

\section{S. Frixione ${ }^{\dagger}$}

\section{$L A P P$}

Chemin de Bellevue, BP 110, 74941 Annecy-le-Vieux CEDEX - France

E-mail: 'Stefano.Frixione@cern.ch'

\section{F. Maltoni}

Department of Physics, University of Illinois at Urbana-Champaign

Urbana, IL 61801, USA

E-mail: maltoni@uiuc.edui

\section{C.R. Schmidt}

Department of Physics and Astronomy, Michigan State University

East Lansing, MI 48824, USA

E-mail: 'schmidt@pa.msu.edu'

ABSTRACT: In this talk we give a short review of forward jets and forward $W$-boson production at hadron colliders, in view of the extraction of footprints of BFKL physics. We argue that at Tevatron energies, dijet production at large rapidity intervals is still subasymptotic with respect to the BFKL regime, thus the cross section is strongly dependent on the various cuts applied in the experimental setup. In addition, the choice of equal transverse momentum cuts on the tagging jets makes the cross section dependent on large logarithms of non-BFKL origin, and thus may spoil the BFKL analysis. For vector boson production in association with two jets, we argue that the configurations that are kinematically favoured tend to have the vector boson forward in rapidity. Thus $W+2$ jet production lends itself naturally to extensions to the high-energy limit.

${ }^{*}$ Speaker.

${ }^{\dagger}$ On leave from INFN, Sez. di Genova, Italy 
In strong-interaction processes characterised by two large and disparate energy scales, which are typically the squared parton center-of-mass energy $\hat{s}$ and momentum transfer $\hat{t}$, with $\hat{s} \gg \hat{t}$, the BFKL theory [i] resums the large logarithms $\ln (\hat{s} /|\hat{t}|)$. Over the past years several attempts have been made to predict and detect footprints of emission of BFKL gluon radiation in strong-interaction processes, like in dijet production at hadron colliders at large rapidity intervals, in forward jet production in DIS and in $\gamma^{*} \gamma^{*}$ collisions in doubletag events, $e^{+} e^{-} \rightarrow e^{+} e^{-}+$hadrons. Here we shall review first dijet production at hadron colliders at large rapidity intervals, and then consider the production of a forward $W$-boson in association with two jets.

\section{Dijet production at large rapidity intervals}

In dijet production at hadron colliders, at large enough rapidities, the rapidity interval is well approximated by the expression $\Delta y \simeq \ln (\hat{s} /|\hat{t}|)$, where $\hat{s}=x_{a} x_{b} S$ and $|\hat{t}| \simeq k_{a \perp} k_{b \perp}$, with $k_{a, b \perp}$ being the moduli of the transverse momenta of the two jets, $x_{a, b}$ the momentum fractions of the incoming partons, and $\sqrt{S}$ the hadronic centre-of-mass energy. Once the transverse momenta are fixed, there are two ways of increasing $\Delta y$ : by increasing the $x$ 's in a fixed energy collider; or viceversa, by fixing the $x$ 's and letting $S$ grow, in a ramping run collider experiment. The former set-up, the only feasible at a collider run at fixed energy, has been proven to be unpractical, since in the dijet production rate $d \sigma / d \Delta y$ as a function of $\Delta y$ it is difficult to disentangle the BFKL-driven rise of the parton cross section from the steep fall-off of the parton densities [20, $]$. The latter set-up, even though the first to be proposed [3] at different centre-of-mass energies. Here we review first the original Mueller-Navelet proposal [풀.

In the high-energy limit, $\hat{s} \gg|\hat{t}|$, any QCD scattering process is dominated by gluon exchange in the crossed channel. This constitutes the leading term of the BFKL resummation. The corresponding QCD amplitude factorizes into an effective amplitude formed by two scattering centres, the impact factors, connected by the gluon exchanged in the crossed channel. The BFKL equation then resums the leading logarithmic (LL) corrections, of $\mathcal{O}\left(\alpha_{S}^{n} \ln ^{n}(\hat{s} /|\hat{t}|)\right)$, to the gluon exchange in the crossed channel. In dijet production at large rapidity intervals, one can write the cross section in the following factorized form [3i]

$$
\frac{d \sigma}{d x_{a}^{0} d x_{b}^{0}}=\int d k_{a \perp}^{2} d k_{b \perp}^{2} f_{\mathrm{eff}}\left(x_{a}^{0}, \mu_{F}^{2}\right) f_{\mathrm{eff}}\left(x_{b}^{0}, \mu_{F}^{2}\right) \frac{d \hat{\sigma}_{g g}}{d k_{a \perp}^{2} d k_{b \perp}^{2}}
$$

where $\mu_{F}$ is the factorization scale, and the effective parton distribution functions (p.d.f.) are [יi=1] $f_{\text {eff }}(x)=G(x)+\frac{4}{9} \sum_{f}\left[Q_{f}(x)+\bar{Q}_{f}(x)\right]$, where the sum runs over the quark flavours, and we understand a dependence of the p.d.f. also on the factorization scale. $x_{a}^{0}, x_{b}^{0}$ are the parton momentum fractions in the high-energy limit,

$$
x_{a}^{0}=\frac{k_{a \perp}}{\sqrt{S}} e^{y_{a}} \quad x_{b}^{0}=\frac{k_{b \perp}}{\sqrt{S}} e^{-y_{b}}
$$


where $y_{a}\left(y_{b}\right)$ is the rapidity of the most forward (backward) jet. In the high-energy limit,

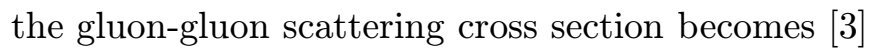

$$
\frac{d \hat{\sigma}_{g g}}{d k_{a \perp}^{2} d k_{b \perp}^{2}}=\left[\frac{3 \alpha_{S}}{k_{a \perp}^{2}}\right] f\left(-k_{a \perp}^{2}, k_{b \perp}^{2}, \Delta y\right)\left[\frac{3 \alpha_{S}}{k_{b \perp}^{2}}\right] \text {. }
$$

with $\Delta y=y_{a}-y_{b} \geq 0$. The quantities in square brackets are the impact factors for jet production. The function $f$ is the solution of the BFKL resummation.

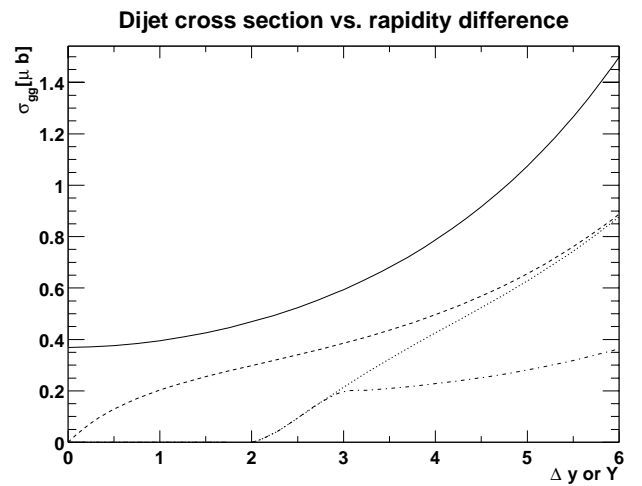

Figure 1: The dependence of the LL BFKL gluon-gluon cross section on $\Delta y$ in the standard Mueller-Navelet calculation (upper solid line) and on $Y$ for the D0 setup. Three curves are shown for the definition of $x$ 's applied in the D0 analysis: dashed line for the requirement $\Delta y>0$, dotted line for $\Delta y>2$, dash-dotted

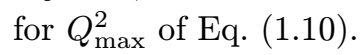

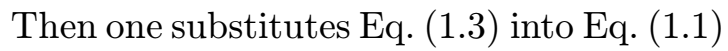
and integrates it over the transverse momenta $k_{a \perp}$ and $k_{b \perp}$ above a threshold $E_{\perp}$, at fixed coupling $\alpha_{S}$ and fixed $x_{a}^{0}, x_{b}^{0}$. The rapidity interval $\Delta y$ is determined from the $x$ 's (1) (1)

$$
\Delta y=\ln \frac{x_{a}^{0} x_{b}^{0} S}{k_{a \perp} k_{b \perp}}
$$

and since it depends on $k_{a \perp} k_{b \perp}$, it is not a constant within the integral. However, the dominant contribution to Eq. (1) the largest value of $\Delta y$, which is attained at the transverse momentum threshold, thus in Ref. [3] $\Delta y$ is fixed at its maximum by reconstructing the $x$ 's at the kinematic threshold for jet production and setting them in a oneto-one correspondence with the jet rapidities

$$
x_{a}^{\mathrm{MN}}=\frac{E_{\perp}}{\sqrt{S}} e^{y_{a}} \quad x_{b}^{\mathrm{MN}}=\frac{E_{\perp}}{\sqrt{S}} e^{-y_{b}} .
$$

Then the factorization formula $\left(\begin{array}{l}1 \\ 1\end{array} \overline{1}_{1}^{\prime}\right)$ is determined at fixed $x_{a}^{\mathrm{MN}}, x_{b}^{\mathrm{MN}}$, and the integration over $k_{a \perp}$ and $k_{b \perp}$ can be straightforwardly performed. Asymptotically, the gluon-gluon cross section becomes

$$
\hat{\sigma}_{g g}^{(\Delta y \gg 1)}\left(k_{a, b \perp}>E_{\perp}\right)=\frac{9 \pi \alpha_{S}^{2}}{2 E_{\perp}^{2}} \frac{e^{A \Delta y}}{\sqrt{\pi B \Delta y / 4}},
$$

with $A=12 \ln 2 \alpha_{S} / \pi$ and $B=42 \zeta(3) \alpha_{S} / \pi$. At very large rapidities the resummed gluongluon cross section grows exponentially with $\Delta y$ (Fig. 11, solid line), in contrast to the LO $\left(\mathcal{O}\left(\alpha_{s}^{2}\right)\right)$ cross section, which is constant at large $\Delta y$. In Eq. (1).1i), the parton momentum fractions being basically fixed, one can interpret a rise of the jet cross section as a function of $\Delta y$ as due to a rise in the parton cross section. Then from the asymptotic formula (i. 6 i) the effective BFKL intercept $\alpha_{B F K L} \equiv A+1$ can be derived.

The D0 Collaboration [4] has revisited the original Mueller-Navelet proposal, and has measured the ratio

$$
R=\frac{\sigma\left(\sqrt{S_{A}}\right)}{\sigma\left(\sqrt{S_{B}}\right)}
$$


of dijet cross sections obtained at two different centre-of-mass energies, $\sqrt{S_{A}}=1800$ $\mathrm{GeV}$ and $\sqrt{S_{B}}=630 \mathrm{GeV}$. The dijet events have been selected by tagging the most forward/backward jets in the event, and the cross section is measured as a function of the momentum transfer, defined as $Q^{2}=k_{a \perp} k_{b \perp}$, and of the quantities

$$
x_{1}=\frac{2 k_{a \perp}}{\sqrt{S}} e^{\bar{y}} \cosh (\Delta y / 2), \quad x_{2}=\frac{2 k_{b \perp}}{\sqrt{S}} e^{-\bar{y}} \cosh (\Delta y / 2),
$$

with $\bar{y}=\left(y_{a}+y_{b}\right) / 2 . \quad x_{1}$ and $x_{2}$ are reconstructed from the tagged jets using Eq. (1) (1) irrespective of the number of additional jets in the final state. In LO kinematics, $x_{1}$ and $x_{2}$ are the momentum fractions of the incoming partons. Higher-order corrections imply that these equalities no longer hold; however, Eq. (1. $\left.\overline{1}_{-} . \bar{l}\right)$ is still a reasonable approximation, except near the borders of phase space. Thus when the ratio in Eq. (I. $\left.\bar{I}_{-} \overline{7}_{i}\right)$ is computed at fixed $x_{1}$ and $x_{2}$, the contributions due to the p.d.f.'s cancel to a large extent, allowing so the study of BFKL effects without any contamination from long-distance phenomena. Then, if Eq. (1, (1)

In the analysis performed by D0 [i] nets have been selected by requiring $k_{a, b \perp}>$ $20 \mathrm{GeV},\left|y_{a, b}\right|<3$, and $\Delta y>2$, and a cut on the momentum transfer, $400<Q^{2}<1000 \mathrm{GeV}^{2}$, has been imposed. These cuts select dijet events at large rapidity intervals.

In the D0 setup there are, however, two main differences with respect to the standard Mueller-Navelet analysis, in which it is assumed that the $x$ 's are reconstructed through Eq. (1).5in) and that the jet transverse momenta are unbounded from above:

a) D0 collect data with an upper bound on $Q^{2}=k_{a \perp} k_{b \perp}$, which is of the same order of magnitude as the square of the lower cut on the jet transverse momenta, and thus cannot be ignored in the integration over the transverse momenta;

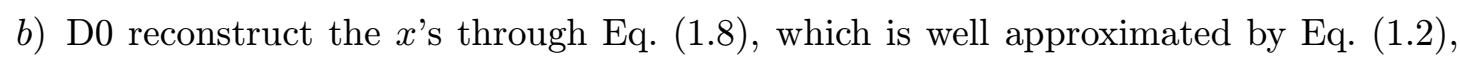
but not by Eq. (1, (1.5i).

Following Ref. [6] section. First, we note that when we use Eq. (1.1. in a given $\left(x_{a}^{0}, x_{b}^{0}\right)$ bin all the transverse momenta and rapidities contribute which fulfil Eq. (1.1.2). Thus the rapidity interval between the jets cannot be used as an independent, fixed observable. For convenience, we write the rapidity interval (1)

$$
\Delta y=Y+\ln \frac{E_{\perp}^{2}}{k_{a \perp} k_{b \perp}},
$$

with $Y=\ln \left(x_{a}^{0} x_{b}^{0} S / E_{\perp}^{2}\right)$. The requirement that the rapidity interval be positive, $\Delta y \geq 0$, imposes an effective upper bound on $Q^{2}: Q_{\max }^{2}=E_{\perp}^{2} e^{Y}$. Integrating then the gluon-gluon cross section (1.3.

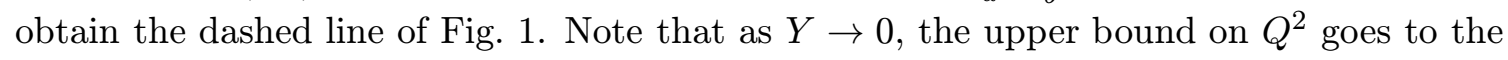
kinematic threshold, $Q_{\max }^{2} \rightarrow E_{\perp}^{2}$, and accordingly the cross section vanishes. When we include the D0 experimental cuts on the transverse momentum, $Q^{2}<1000 \mathrm{GeV}^{2}$, and the 
rapidity interval, $\Delta y \geq 2$, the same analysis can be repeated except that the upper bound on $Q^{2}$ is given by

$$
Q_{\max }^{2}=\min \left(1000 \mathrm{GeV}^{2}, E_{\perp}^{2} e^{(Y-2)}\right),
$$

where we have used the fact that $\Delta y>2$ imposes the second effective upper bound on $Q^{2}$. Integrating then the gluon-gluon cross section (1-3i) over $k_{a \perp}$ and $k_{b \perp}$, we obtain the dot-dashed line of Fig. i i Thus the shape of the cross section as a function of $Y$ depends crucially on the upper bound on $Q^{2}$. Asymptotically [6] the same scaling with $Y$ as Eq. (1) (1) signals that within the D0 kinematic regime we are far from the asymptotic region. Care must therefore be taken in interpreting any observed cross section increase in the D0 data as due exclusively to the BFKL intercept.

In addition, the analysis of dijet production according to the D0 acceptance cuts

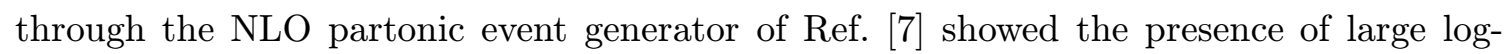
arithms of non-BFKL origin [i, i $]$, due to the choice of equal transverse momentum cuts, $k_{a \perp}, k_{b \perp}>E_{\perp}$. Such logarithms are bound to affect the BFKL analysis, but can be easily avoided if different cuts on the transverse momenta of the two jets are chosen. Accordingly,

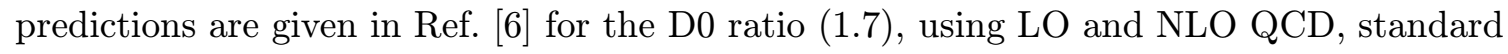
("naive") BFKL, and BFKL with energy-momentum conservation and running coupling

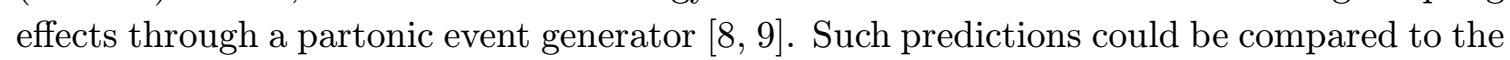
D0 data, if these are re-analysed with different transverse momentum cuts on the tagging jets.

\section{Forward $W$ boson production with associated jets}

In the previous section, we have reviewed the feasibility of extracting footprints of the BFKL resummation through the Mueller-Navelet analysis of forward dijet production; we mentioned, but dismissed summarily, dijet production at a fixed energy collider because an eventual BFKL-driven rise of the parton cross section within the rate $d \sigma / d \Delta y$, as a function of $\Delta y$, would be hindered by the steep fall-off of the p.d.f.'s. Infact, except at large $x$ 's (i.e. $x \gtrsim 0.1$ ), dijet production is dominated by gluon-gluon scattering, and since the shape of the gluon p.d.f. is very sensitive to $x$ variations (and monotonically decreasing with it), the dijet production rate turns out to be sensitive to the approximation made (e.g. in the BFKL analysis) in reconstructing the $x$ 's from the jet kinematic variables.

In this section, following the analysis of Ref. [i] 1 in $]$, we shall revive the quest for BFKL footprints at a fixed energy hadron collider by considering forward $W$ boson production in association with two jets. We believe to have reasons to prefer forward $W$ boson production in association with two jets to dijet production. Firstly, it might be experimentally easier to pick up forward $W$ bosons that decay leptonically than forward jets; once a forward lepton has triggered the event, one observes the jets that are associated to it, with no limitations on their transverse energy. Conversely, in a pure jet sample one usually triggers the event on a jet of relatively high transverse energy, thus the triggering jet cannot be too forward. Secondly, $W$ production in association with jets lends itself naturally to extensions to the high-energy limit, since it favours configurations with a forward $W$ boson. Presently we 
examine this remark by analysing in detail the kinematics of $W+2$ jet hadroproduction. At LO the parton subprocesses are

$$
\begin{aligned}
& \text { (a) } g g \rightarrow W q \bar{q}, \\
& \text { (b) } q \bar{q} \rightarrow W g g+W q \bar{q}, \\
& \text { (c) } q q \rightarrow W q q, \\
& \text { (d) } q g \rightarrow W q g .
\end{aligned}
$$

The momentum fractions of the incoming partons are given through energy-momentum conservation by

$$
\begin{aligned}
& x_{a}=\frac{k_{j_{1 \perp}}}{\sqrt{s}} e^{y_{j_{1}}}+\frac{k_{j_{2 \perp}}}{\sqrt{s}} e^{y_{j_{2}}}+\frac{m_{\perp}}{\sqrt{s}} e^{y_{W}} \\
& x_{b}=\frac{k_{j_{1 \perp}}}{\sqrt{s}} e^{-y_{j_{1}}}+\frac{k_{j_{2 \perp}}}{\sqrt{s}} e^{-y_{j_{2}}}+\frac{m_{\perp}}{\sqrt{s}} e^{-y_{W}}
\end{aligned}
$$

with $k_{j_{1,2 \perp}}$ the jet transverse momenta and $m_{\perp}=\sqrt{m_{W}^{2}+\left|k_{j_{1 \perp}}+k_{j_{2 \perp}}\right|^{2}}$ the $W$ transverse mass.

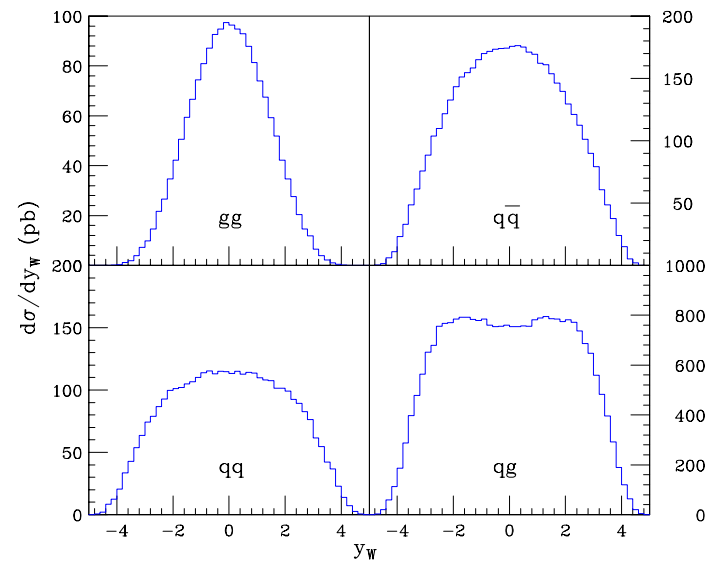

Figure 2: Distributions in $y_{W}$ for the subprocesses of Eq. (2.1i) at the LHC centre-of-mass energy $\sqrt{s}=14 \mathrm{TeV}$ and with $p_{j_{\perp} \min }=30$ $\mathrm{GeV}$.

tends to follow the $W$ in rapidity. The position of the other jet is a dynamical feature peculiar of $W+2$-jet production: thanks to the gluon exchanged in the crossed channel, in $(b),(c)$ and $(d)$ that jet can be easily separated in rapidity from the $W$ boson.

In Fig. 'i $\overline{3}_{1}^{-}$we consider $W+2$-jet production as a function of $\Delta y$, and with acceptance cuts $y_{W}, y_{j_{2}} \geq 1$ and $y_{j_{1}} \leq-1$, or $y_{W}, y_{j_{2}} \leq-1$ and $y_{j_{1}} \geq 1$, namely we put a veto on tagging jets in the central rapidity region. Note that Fig. $\overline{3}$ is both qualitatively and quantitatively different from $d \sigma / d \Delta y$ in dijet production [2i]: the peak is a striking confirmation of the dominance of the configurations asymmetric in rapidity, discussed above. In fact the veto in the central rapidity region strongly penalises the asymmetric configurations when $\Delta y$ approaches its minimum value; since the asymmetric configurations dominate the $W+2$-jet 


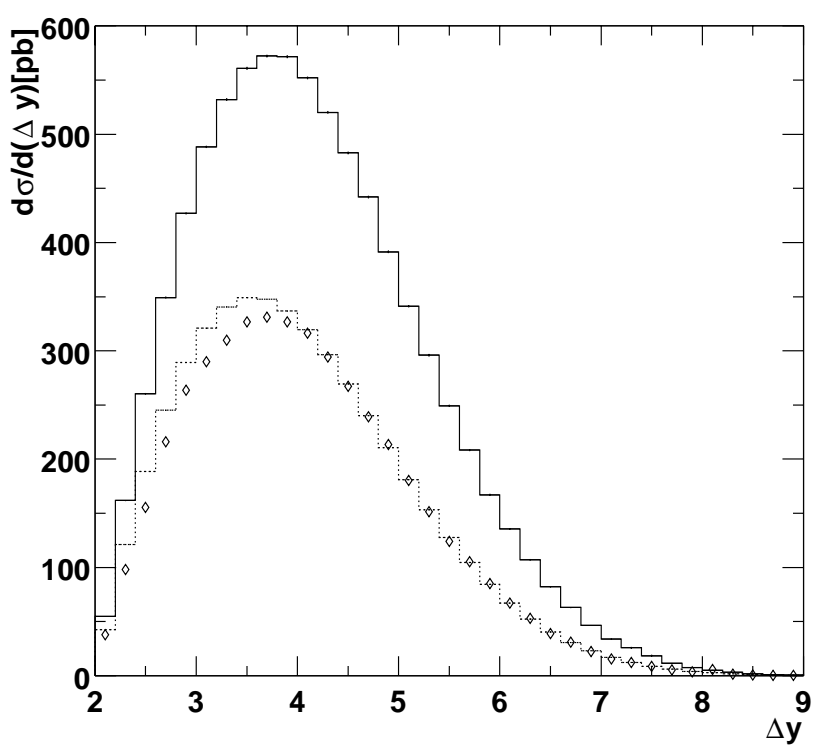

Figure 3: The $W+2$-jet production rate as a function of the rapidity interval between the jets $\Delta y=\left|y_{j_{1}}-y_{j_{2}}\right|$, with acceptance cuts $y_{W}, y_{j_{2}} \geq 1$ and $y_{j_{1}} \leq-1$, or $y_{W}, y_{j_{2}} \leq-1$ and $y_{j_{1}} \geq 1$. The diamonds are the exact production rate; the dashed-dotted curve is the production rate in the highenergy limit; the solid curve includes the BFKL corrections and energy-momentum conservation through a BFKL partonic event generator.

production rate, the effect is a strong depletion of the latter. In addition, the BFKL ladder, which includes energy-momentum conservation, shows a substantial increase of the cross section with respect to the LO analysis, as opposed to the decrease of the dijet case $[9 \overline{9}]$. Infact, the implementation of energy-momentum conservation in the BFKL partonic event generator has a much lesser impact than in the dijet case. This is due to the fact that the valence quark distribution in $q g \rightarrow q g W$ is much less sensitive to $x$ variations than the gluon distribution in $g g \rightarrow g g$. Secondly, the presence of at least three particles in the final state makes the threshold configurations, and thus the logarithms of non-BFKL origin, much less compelling than in the dijet case.

The analysis above lets us hope that $W+2$ jet production at the LHC will turn out to be a good test in favour of, or against, the presence of the BFKL resummation in hadron collisions.

\section{References}

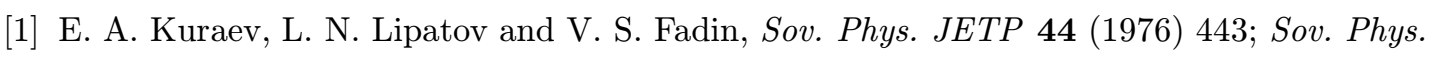
: - JETP $45(1977) 199$

I. I. Balitsky and L. N. Lipatov, SSov. J. Nucl. Phys. 28 (1978) 822

[2] V. Del Duca and C. R. Schmidt, 'Phys. Rev. D 49 (1994) 4510; [hep-ph/931129ol];

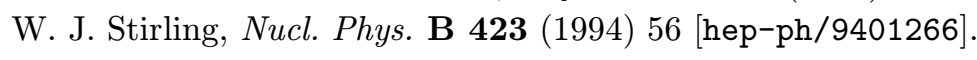

[3] A. H. Mueller and H. Navelet, iNucl. Phys. B 282 (1987) 727 i 
[4] B. Abbott et al. [D0 Collaboration], Physs. Rev. Lett. 84 (2000) 5722 [hepex-ex/9912032].

[5] B. L. Combridge and C. J. Maxwell, 'Nucl. Phys. B 239 (1984) 429 i.

[6] J.R. Andersen, V. Del Duca, S. Frixione, C. R. Schmidt and W. J. Stirling,

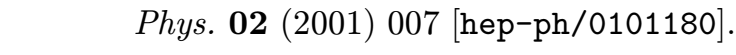

[7] S. Frixione, №cl. Phys. B 507 (1997) 295 inep-ph/9706545i.

[8] C. R. Schmidt, Phys. Rev. Lett. 78 (1997) 4531 [hep-ph/9612454].

[9] L. H. Orr and W. J. Stirling, 'Phys. Rev D 56 (1997) 5875 l'hep-ph/9706529i];'Phys. Lett. -

[10] J.R. Andersen, V. Del Duca, F. Maltoni and W. J. Stirling, U. High Energy Phys. 05 (2001)i 048 [hep-ph/0105146]. 